\title{
THE GAIT ANALYSIS OF PREGNANT WOMEN
}

\author{
Tsan-Hsun Huang', Sheng-Che Lin ${ }^{2}$, Chin-Shan Ho' ${ }^{\prime}$ Chia-Yuen Yu', You-Li Chou ${ }^{\prime}$ \\ ${ }^{1}$ Institute of Biomedical Engineering, National Chung Kung University, and \\ ${ }^{2}$ Section of Plastic Surgery, Department of Surgery, National Cheng Kung \\ University Medical Center, Tainan, Taiwan
}

\begin{abstract}
During pregnancy, a woman changes obviously in body weight, body shape, and endocrine system. Those changes make the posture and gait pattern of the pregnant women different from those of the non-pregnant women. At the same time, activities of daily living become more difficult. But there was still no published paper discussing the posture and gait changes of the pregnant women in details and in a whole.

In this study, we used the optimization method to define the hip joint center of the pregnant women. The optimization method could compensate the lack of the anthropometric data of the pregnant women. Due to the variations of physiological changes in the pregnant women, it was difficulty to standardize the gait analysis of pregnant women, even in the same gestational age. The results revealed that there really was some correlation between the significant finding of gait analysis and sacroiliac pain of the pregnant women. The sacroiliac pain often occurred in the following conditions of gait analysis: (1) when the hip extension moment increased; (2) when the knee extension moment decreased; (3) when the angle of ankle plantar-flexion decreased; and (4) when the sacroiliac ajoint torsion increased. The gait analysis of the pregnant is a feasible, but complicated method for study. The data of gait analysis were also compared with questionnaires for further analysis.
\end{abstract}

Biomed Eng Appl Basis Comm, 2002 (April): 14: 67-70.

Keywords: pregnant women, gait analysis, sacro-iliac pain

\section{INTRODUCTION}

There are many physiological changes during pregnancy, especially in body weight, body shape, and endocrine system. Those changes make the pregnant women different from the non-pregnant women in posture and gait pattern. At the same time, activities of daily living become more difficult [1]. The problems needed be solved. But there was still no published paper discussing the posture and gait changes of the pregnant women in details and in a whole.

The increases in body weight and body mass are

Received: Dec. 04, 2001; Accepted: March 05, 2002

Correspondence:You-Li Chou, Ph.D., Professor

Institute of Biomedical Engineering, National Chung Kung University.

No 1, Ta-Hsueh Road, Tainan 70148, Taiwan.

E mail:ylchou@mail.ncku.edu.tw mainly distributed over the trunk and pelvic area. These create loads on the spine, make body alignment much different, and also increase the moment of inertia in the pelvis. The posture is changed with increasing lumbar lordosis, thoracic kyphosis, and backward leaning of head. So, it takes much more time for the pregnant women to change postures (Fig. 1) [2]. In the meanwhile, the "relaxin" in serum affects the relaxity of all joints, especially in the pelvis. It makes the situation of the hip joint more loosening and makes the posture and gait pattern different [3]. The body fluid increases during pregnancy [4] when the pregnant woman gets weight. The increasing body fluid is mainly distributed over the hip joint, and the moment of inertia increases [5].

More than $48-56 \%$ of the pregnant women have complaint of back pain [6-8]. Back conditions of the pregnant women might improve after the "back pain school" course [9]. But the course of the exercise is still uncertain and shall be individualized [10]. 


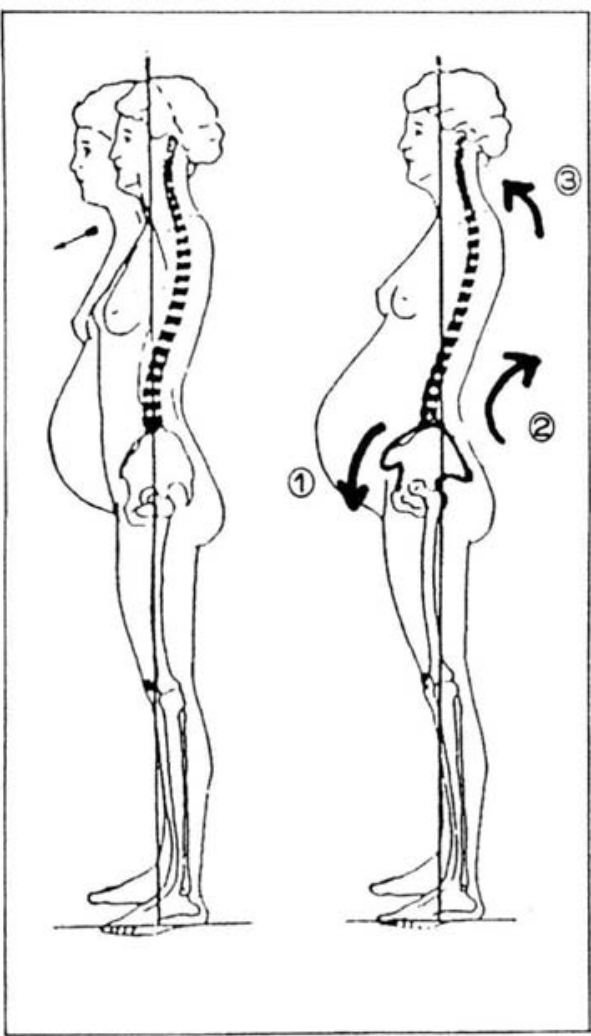

Fig. 1 The postural changes of spine angle during preguancy.

In this study of motion analysis of pregnant women, the most difficult problem is the lack of the anthropometric data. We have to find a reasonable model to compensate this situation. Researchers choose the hip joint, which is the major change during the pregnancy, to be defined by the optimization method.

\section{MATERIALS AND METHODS}

There were ten pregnant women participated in this study. And another ten women without pregnant experience were also included as control group. The pregnant women were divided into three groups: (1) Initial stage- -within 12 gestational weeks, (2) midterm stage-13- 28 gestational weeks, (3) late stage--29-40 gestational weeks. There were 10 normal data and 20 experimental data. Every data included better 5 trials for average.

At first, the pregnant subject fills the questionnaires: The Pain and Impairment Relationship Scale (PAIRS) and Body Part Discomfort Rating Scale (BPDRS)[15]. Then, the Helen Hayes markers system was put on the subject, and level walking in natural pattern was collected by EVA motion analysis system (Motion Analysis Corporation, Santa Rosa, California, USA).
The processes were as follows:

1. Use Optimization method to define the hip joint center: We hypothesized that the leg swing was a global movement. The optimized equation is as follows:

$$
f=\sum_{1}^{n}\left[\left(x_{1}-a\right)^{2}+\left(y_{1}-b\right)^{2}+\left(z_{1}-c\right)^{2}-(\text { leg-length })^{2}\right]
$$

$(\mathrm{a}, \mathrm{b}, \mathrm{c})$ : the hip joint center;

leg-length: leg length;

" $i$ " : the duration of swing phase

2. Compare the optimized data with the origin data operated by OT4 software.

3. Compare the pregnant data with the normal data.

\section{RESULTS AND ANALYSIS}

The gait pattern of normal woman after optimization was quite similar to that of the same subject operated by OT4 software in joint angles, moment, and power of hip, knee and ankle joint (Fig. 2). So we extended to use this optimization method to decide the hip joint center of pregnant women.

When comparing the pregnant data after optimization with the same subject operated by OT4 software, these results revealed significant differences existed, especially in knee abduction angle, knee and hip internal rotation angles, hip extension moment, and hip power (Fig. 3). We think the optimization is an important and reasonable process.

When comparing the data of the pregnant in different gestational stages, the results showed four significant differences in (1) increases of hip extension moment; (2) decreases of knee extension moment; (3) decreases of ankle plantar flexion moment and (4) increases of knee adduction moment (Fig. 4).

\section{DISCUSSIONS}

Generally, we think the reason of the pregnant back pain is the increase of lumbar lordosis [10]. But actually the sacroiliac pain is more often occurred than lower back pain [11] during pregnant. Hassan (1985) doesn't think that the back pain of the pregnant is due to lordosis. Ostgaard (1993) and Franklin (1998) find that lumbar lordosis is increasing during pregnancy, but there is no significant relationship between increasing lordosis and back pain of the pregnant. They suggest the dynamic motion study maybe a good direction to find the reasons of pregnant back pain [12-14]. Our results revealed that Joint angles, moment, and the power curves were almost the same between the optimization method and the commercial OT4 methods. 

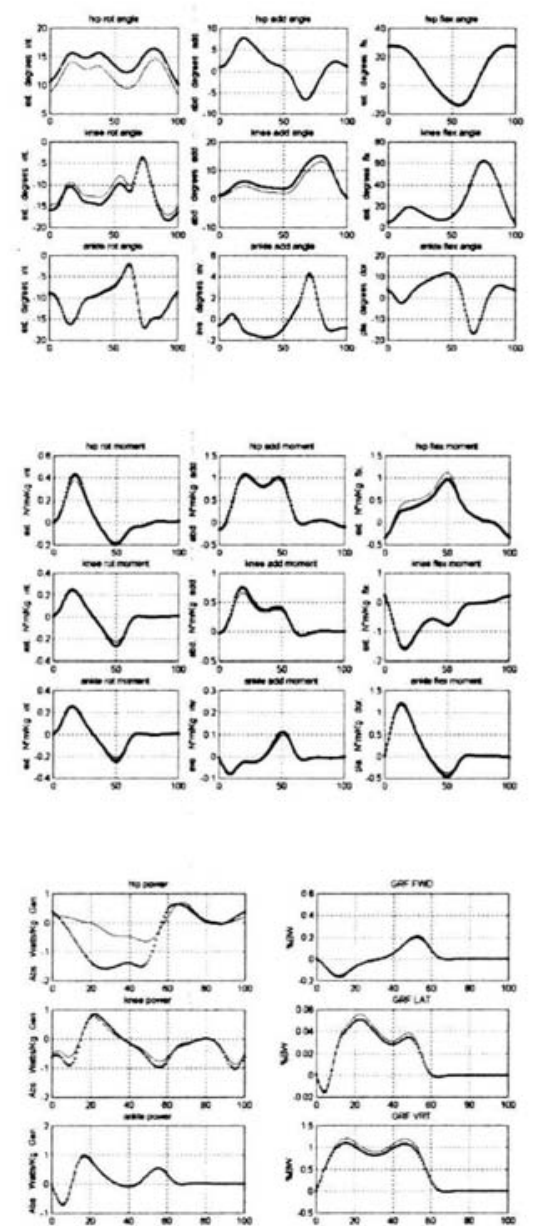

Fig. 2 The angles, moments and powers of three joints (hip, knee, ankle) in both normal subject data operated by OT 4 software and that after optimizatio

And there were some significant differences between the pregnant and normal subjects, especially in knce abduction angle, knee and hip internal rotation angles, hip extension moment, and hip power. Because of these differences, we thought the optimization is a very important process.

We found the hip moment of pregnant women increased and other joints' movement and moment decreased. In the questionnaires, the pregnant subjects often complained back pain, especially sacroiliac pain. We found this pain was related to the following conditions:

(1) Increasing hip extension moment

(2) Decreasing knee extension moment

(3) Decreasing plantar flexion moment

(4) Increasing knee adduction moment

Because of these conditions, the hip is the main work-loading area. Otherwise, the anterior tilt of pelvis makes the hip extensor kept lengthening, then the hip
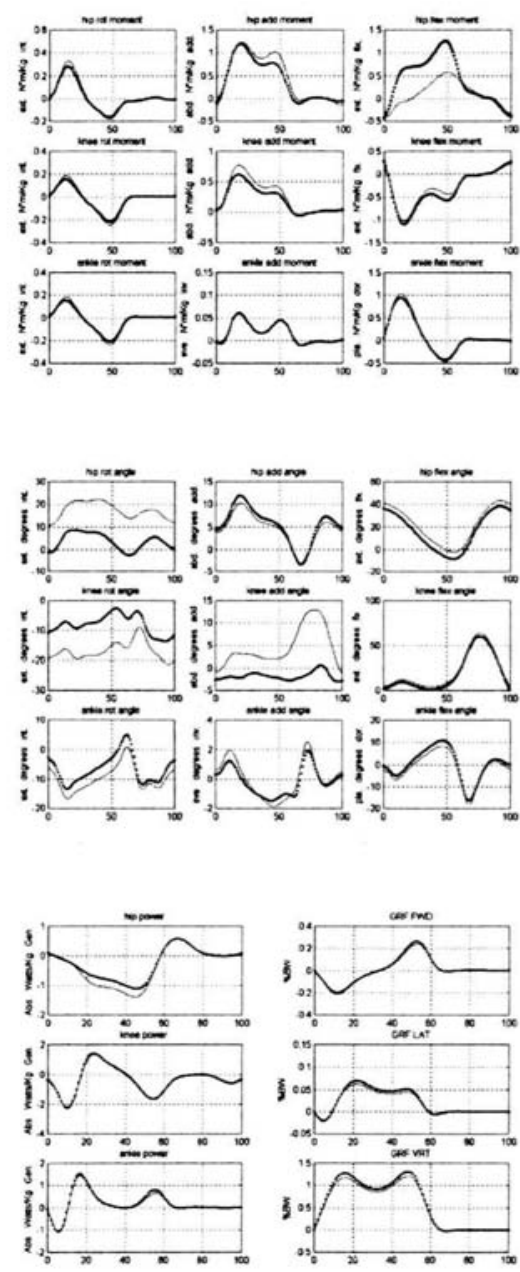

Fig. 3 Comparison of angles, moments and powers in three joints (hip, knee, ankle) between the pregnant data after optimization (dot line) with the data of same subject operated by OT 4 software (black line).

extensors are over stretched and easily fatigue. That is highly related to the sacroiliac pain of the pregnant women.

\section{CONCLUSIONS}

In motion analysis of pregnant women, there were significant changes in the gait and posture. And we used the optimization method to compensate the lack of the pregnant anthropometric data. In this study, we found that gait analysis of pregnant women with sacroiliac pain revealed significant increases in hip extension moment and knee adduction moment, and significant decreases in knee extension moment and ankle plantar flexion moment. 

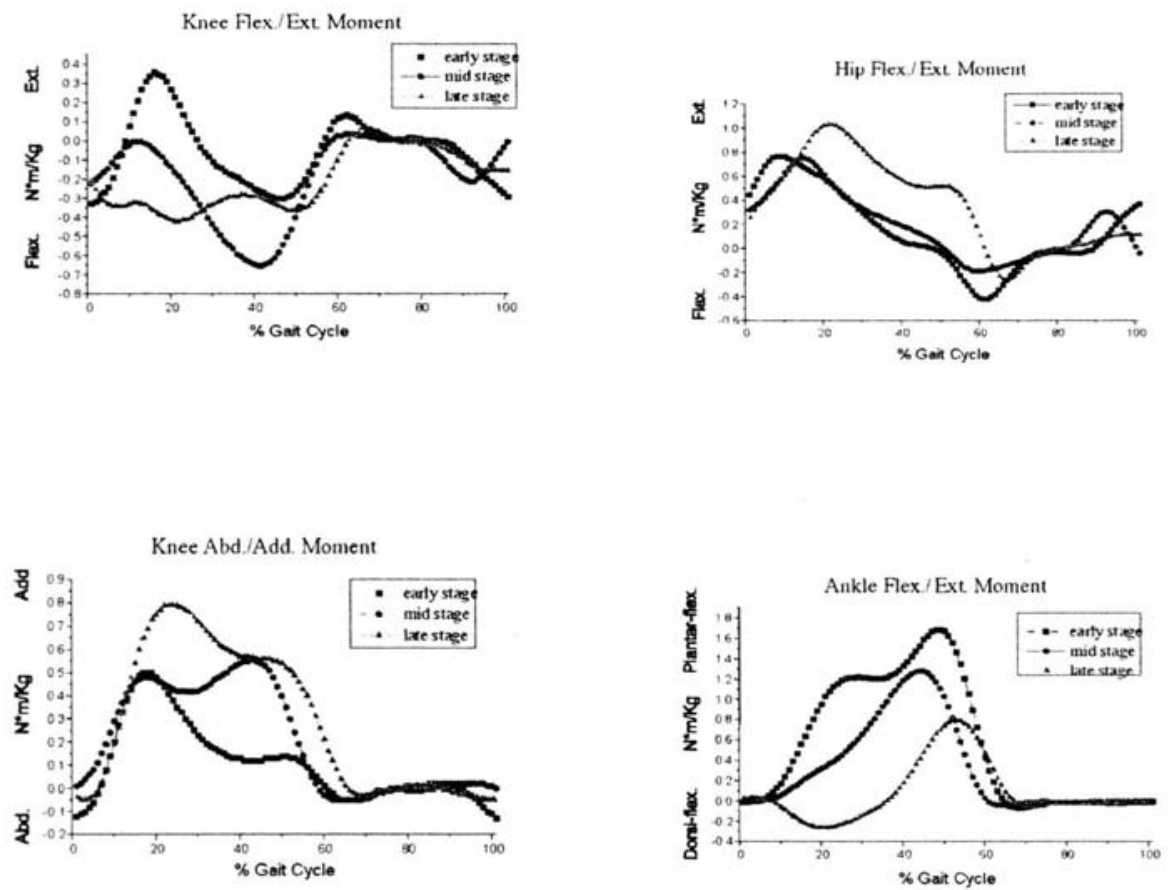

Fig. 4 The moment of the pregnant's hip, knee and ankle joints in different gestational stages (late stage: faint black; mid-term stage: moderate black; initial stage: dark black).

\section{REFERENCES}

1. Nichollo J.A., Grieve D.W.: Performance of Physical Tasks in Pregnancy. Ergnonomics 35, 301-311, 1992.

2. Bookhout M.M., Boissonault W.G.: Physical Therapy Management of Musculoskeletal Disorders during Pregnancy. In: E. Wilder (Ed.), Obstetric and gynecology physical Therapy, ChurchillLivingstone, In pregnancy: hypothetical physiological mechanisms. Am J Phys Med Rehab. 68:227-229,1979.

3. MacFayden I.F.: Maternal Aspects of Pregnancy. In: Livingstone, Edinburgh, 151-172, 1989

4. Abrams B., Parker J.D.: Maternal Weight Gain in Women with Good Pregnancy Outcome. Obstet Gynecol. 76, 1-7, 1990.

5. Jensen R.K., Doucet S., Treitz T.: Changes in Segment Mass and Mass Distribution during Pregnancy. J Biomechanics. 29(2):251-256,1996.

6. Mantle M.J., Greenwood R,M., Curry H.L.F.: Back Ache and Pregnancy. Rheumatol Rehabil. 16:95$101,1977$.

7. Fast A., Shapiro D., Ducommun E.J. et al.: Low
Back Pain in Pregnancy. Spine. 12: 368-371, 1987.

8. MacEvilly M., Bulgy D.: Back Pain and Pregnancy: A Review. Pain. 64:405-414, 1996.

9. Wang T.W., Apgar B.A.: Exercise during Pregnancy. American Family Physician.57: 1846-1852, 1998.

10. Moore K., Dumas G.A., Reid J.G.: Postural Changes Associated with Pregnancy and their Relationship with Low Back Pain. Clin Biomech. 5: 169-174, 1990.

11. Ostgaard H.C., Andersson G.B., karlsson K.: Prevalence of Back Pain in Pregnancy. Spine. 16(5): 549-52, 1991.

12. Hasson T., Bigoss B.P., Wortley M. The Lumbar Lordosis in Acute and Chronic Low Back Pain. Spine. 10:154-155, 1985.

13. Ostgaard H.C., Andersson G.B.J., Schultz A.B., Miller J.A.: Influence and Some Biomechanical Factors on Low Back Pain in Pregnancy. Spine. 18:61-65, 1993

14. Mary E., Franklin, and Teresa C.K.: An Analysis of Posture and Back Pain in the First and Third Trimesters of Pregnancy. J Orthop Sports Phys Therap. 28:133-138, 1998.

15. Riley J. et al.: Chronic Pain and Functional Impairment: Assessing the Results about Their Relationship. A.P.M.R. 69: 579-582, 198 